\title{
A case of primary hypoadrenalism secondary to amyloidosis
}

\author{
John Watkins ${ }^{1}$, Alice Verran ${ }^{1}$, Milan Piya ${ }^{1,2}$, Saboor Aftab ${ }^{1,2}$, Harpal S Randeva ${ }^{1,2}$, Yen Yeo², \\ Thomas M Barber ${ }^{1,2}$, Narendra L Reddy ${ }^{1,2}$ \\ Warwick Medical School'1, University of Warwick, Clinical Sciences Research Laboratories, Coventry, UK \\ University Hospitals of Coventry \& Warwickshire ${ }^{2}, U K$
}

\section{Introduction}

Endocrinopathy is a rare complication of systemic amyloidosis and can involve a number of tissues and organs including the adrenal glands [1]. We report a case of primary adrenal failure secondary to systemic light chain amyloidosis (AL). AL amyloidosis is the most common form of systemic amyloidosis and is associated with plasma cell dyscrasia leading to extra-cellular deposition of fibrilforming monoclonal immunoglobulin light chains, usually of the lambda isotype [2]. Due to its systemic involvement, it has many manifestations which can be vague and difficult to recognize.

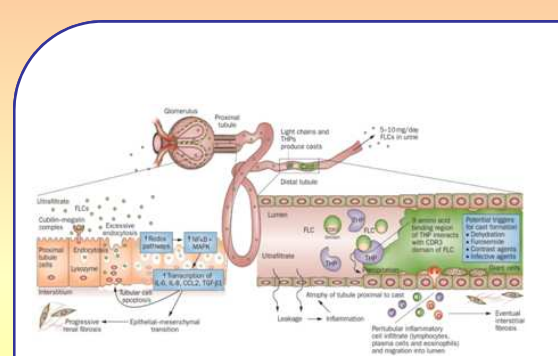

Figure 1a

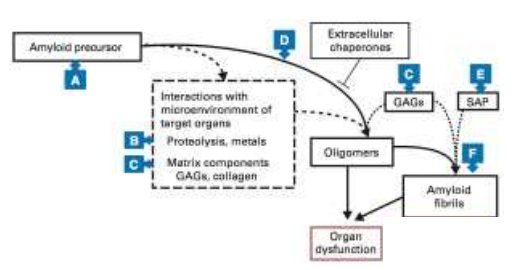

Figure 1b
Figure 1a: Mechanism of free light-chain induced injury Figure 1b: Cascade of molecular events leading to amyloidosis

\section{Case}

A 42-year-old Somalian lady presented with a two year history of lethargy, febrile episodes, and weight loss of over twenty one kilograms. Investigations showed raised serum lambda light chain $103 \mathrm{mg} / \mathrm{L}$ (5.7-26.3), paraproteinaemia $(7 \mathrm{~g} / \mathrm{L})$, and $8 \%$ plasma cells on bone marrow aspirate. A diagnosis of lambda light chain secreting plasma cell dyscrasia was made. She subsequently developed renal failure and renal biopsy confirmed AL amyloidosis. CTDa chemotherapy (attenuated cyclophosphamide, thalidomide, dexamethasone) was initiated. She further developed macroglossia, severe sensorimotor axonal generalised peripheral neuropathy and minor autonomic neuropathy each respectively manifesting as loss of sensation distally, postural hypotension, and altered bowel habit.

Amyloidosis was further confirmed on duodenal biopsy (see figure) and serum amyloid protein (SAP) scintigraphy demonstrated moderate total body amyloid load including liver and spleen.

Two years later, she was admitted following cardiac arrest from neutropaenic sepsis and renal failure requiring external cardio-pulmonary support. Hypotension did not resolve (mean blood pressure: $76 / 44 \mathrm{mmHg}$ ) despite overall recovery through resolution of sepsis and renal failure (haemodialysis).

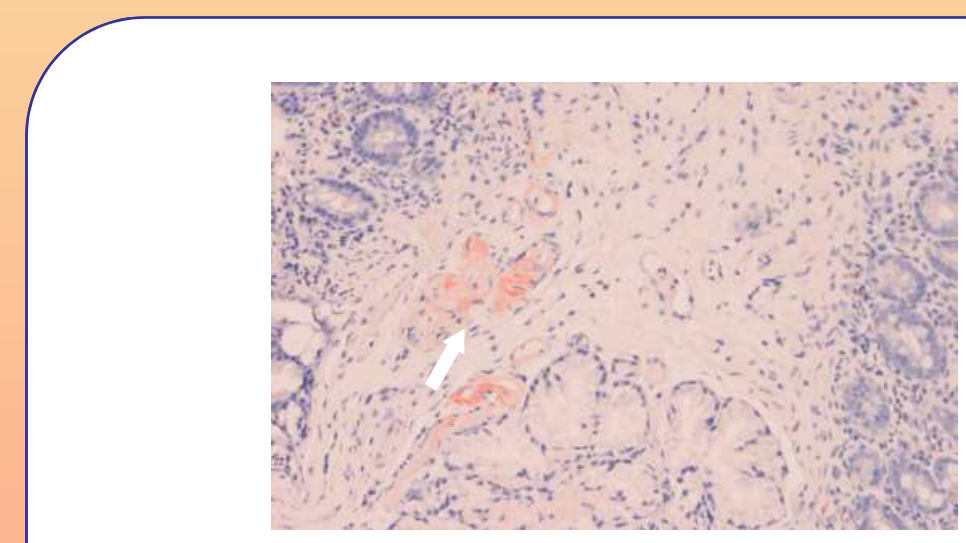

Figure 2: Duodenal biopsy shows amyloid deposition with characteristic salmon pink on Congo red staining. $80 \%$ of biopsies show gastrointestinal involvement, leading to malabsorption.

\section{Management}

Hypoadrenalism was clinically suspected and was confirmed through short synacthen test $(60$ minute cortisol response: $128 \mathrm{nmol} / \mathrm{L})$.

Hypotension resolved (mean blood pressure: $126 / 80 \mathrm{mmHg}$ ) on parenteral hydrocortisone $(400 \mathrm{mg} /$ day) and oral fludrocortisone (50 $\mu \mathrm{g} / \mathrm{day})$.
A normal pituitary scan and an appropriate pituitary biochemical response $\{\mathrm{IGF} 1 \quad 16.3(13-37 \mathrm{nmol} / \mathrm{L})$, LH<1(212iU), FSH<1(2-10iU), TSH $1.05(0.35-6 \mathrm{mU} / \mathrm{L})$, T4 13.5(9$26 \mathrm{pmol} / \mathrm{L})$, post steroid ACTH $17.4(<46.1 \mathrm{ng} / \mathrm{L})$, prolactin $881(<600 \mathrm{mu} / \mathrm{L})\}$ ruled out secondary hypoadrenalism. POEMS syndrome was ruled out based on a normal plasma VEGF level and absence of full clinical spectrum of the syndrome. She was haemodialysis dependent and was stable on oral hydrocortisone and fludrocortisone. She completed six cycles of lenolidamine/dexamethasone for multiple myeloma and eventually died of massive pulmonary haemorrhage.

\section{Learning points}

- Diagnosis of adrenal insufficiency can be challenging in critically ill patients diagnosed with conditions involving multiple organs, such as systemic amyloidosis.

High index of clinical suspicion is needed to prevent this potentially fatal condition, as endocrine dysfunction in systemic amyloidosis is more frequent than it was once thought. 